\title{
Predictive value of neck imaging reporting and data system (NIRADS) in CECT/CEMRI of laryngeal and oral cavity squamous cell carcinoma
}

\author{
Ahmed S. Abdelrahman*, Manar Maamoun Mohamed Ashour and Tougan Taha Abdelaziz
}

\begin{abstract}
Background: The neck imaging reporting and data system (NI-RADS) is a structured reporting algorithm linked with further patient management recommendations. This study was conducted to assess the overall and time point predictive value of the NI-RADS in laryngeal and oral cavity squamous cell carcinoma (SCC) using contrastenhanced magnetic resonance imaging (CEMRI) and contrast-enhanced computed tomography (CECT).

Results: The rate of tumor recurrence was statistically different among the NI-RADS 1-3 categories with recurrence trend for higher NI-RADS scores. The overall negative predictive value (NPV) of the NI-RADS 1 and 2 were 94.3\%, $74.3 \%$ respectively, and the positive predictive value (PPV) of the NI-RADS 3 was $80.8 \%$. The overall recurrence rate of NI-RADS 3 was higher in oral cavity SCC (87.5\%) compared to the laryngeal SCC (70\%). The PPV of overall NIRADS 3 in the follow-up scans (77.8\%) was higher than in the first scan (70.6\%). The odd ratio of tumor recurrence in NI-RADS 3 primary lesion was 19.6.

Conclusion: The predictive value of NI-RADS was significantly different among its categories. Increasing NI-RADS score is associated with increased recurrence among the treated laryngeal and oral cavity SCC. The morphological and enhancement lexicon features equally assign the NI-RADS 3 score.
\end{abstract}

Keywords: NI-RADS, Laryngeal SCC, Oral cavity SCC

\section{Background}

Post-treatment recurrence is detected in $15-50 \%$ of patients with head and neck squamous cell carcinoma (HNSCC) which significantly contribute to disease mortality and morbidity [1-5].

Post-treatment distorted normal anatomical features is challenging for optimal reporting, resulting in variable interpretation, uncertain impressions, vague interobserver agreement, and reports missing further management recommendations. The absence of standardized surveillance protocol for imaging patients with treated

\footnotetext{
*Correspondence: dr_ahmedsamy@yahoo.com

Radiology Department, Faculty of Medicine, Ain Shams University, Cairo, Egypt
}

HNSCC, which varies according to each institute policy,

The structured report is a dynamic process, developed

To overcome these obstacles and to gain the benefit of the structured report, the American College of Radiology (ACR) designed the neck imaging reporting and data system (NI-RADS) as a standardized template, which was linked to management recommendations according to suspicion of recurrence $[8,9]$. The primary and nodal tumor target sites are assigned in a numerical ordinal (1) 
score level from 1 to 4 according to imaging features and level of recurrence suspicion $[10,11]$.

Standardization of the radiological impression with the structured report is the radiological reporting new era trying to give a precise answer for the clinician question and sharing in the patient management by giving the suitable further next step management according to the radiological imaging and suspicious of recurrence.

Although the white paper of the ACR NI-RADS was released in 2018, there are still few published figures of the recurrence rate according to the NI-RADS categories scoring system.

This study aimed to validate the beneficial effect of the NI-RADS system in routine clinical practice and to clarify the predictive value of each NI-RADS category in the treated laryngeal and oral cavity squamous cell carcinoma (SCC) using contrast-enhanced magnetic resonance imaging (CEMRI) and contrast-enhanced computed tomography (CECT).

\section{Methods \\ Patients}

Seventy-four scans of thirty-four patients with mean age $54.5 \pm 2.08$ years were retrospectively evaluated during the period from August 2018 till June 2020. The prerequisite of written consent was waived after the approval of our ethical committee.

According to our institution surveillance protocol, the first baseline scan was performed 8 weeks after finishing treatment and followed by a surveillance follow-up scan at 3-month intervals for 2 years. The inclusion criteria were patients with treated oral cavity and laryngeal SCC who post-treatment surveillance CEMRI or CECT images were interpreted according to the NI-RADS template lexicon with no sex predilection. The exclusion criteria incorporated patients with head and neck cancer other than laryngeal and oral cavity SCC, patients with missed final outcome data, patients with NI-RADS 4 lesion as they have a pathology-proven recurrence as defined by the ACR, non-contrast studies not fulfilling the NI-RADS lexicon, and poor-quality images hinder proper assessment of target lesions.

\section{Technique of contrast-enhanced MRI examination}

- The MRI examination was done using 1.5 T MRI (Philips Achieva scanner, Healthcare, Netherlands) with head and neck coil. The field of view extended from the skull base down to the root of the neck, additional small field of view was taken in cases of oral cavity lesion extending from the sella turcica down to the floor of the mouth and submandibular gland. Sequences: axial DWI, axial T1WIs (TE/TR: 21/633 ms), axial T2WIs (TE/TR: 10/7039 ms), coronal T1 (TE/TR: 14/555 ms), coronal T2 STIR $(\mathrm{TR} / \mathrm{TI}=3500 / 150, \mathrm{TE}=80 \mathrm{~ms})$, and sagittal T2WI (TE/TR: $100 / 3196.7 \mathrm{~ms}$ ). A total of $0.1 \mathrm{mmol} / \mathrm{kg}$ gadolinium was injected at a rate of $2-3 \mathrm{ml} / \mathrm{s}$, then the post-contrast T1WI with fat saturation was obtained in the axial (TR/TE: 611/21 ms), sagittal (TR/ TE: 570/14 ms), and coronal (TR/TE: 570/14 ms) planes. The slice thickness: $4 \mathrm{~mm}$. Scan time: $18-25$ min.

\section{Technique of contrast-enhanced CT examination}

- The CT examination was performed using a multidetector spiral CT, 128-row MDCT scanner (GE 128, Optima 660, USA), the patient was lying in a supine position with his/her arms placed down. An axial image started from the frontal sinuses down through the mediastinum was obtained after injection of 100 milliliters of iodinated contrast agent at a rate of $1-1.5 \mathrm{ml} / \mathrm{s}$ with FOV $=28 \mathrm{~cm}$ and slice thickness of $0.6-0.75 \mathrm{~mm}$. The native images were reformatted with a slice thickness of $3 \mathrm{~mm}$ and transferred to workstations (AW volume share 7 and 3D synapse, Fujifilm) for display using soft tissue window (width 350-400 and level 20-50).

\section{Analysis of data \\ Imaging analysis}

- Forty-six scans for treated laryngeal SCC and 28 scans for treated oral cavity SCC were independently evaluated by two head and neck radiologists with 15 and 11 years of experience, if their diagnosis was discordant, a final diagnosis was made by consensus. $\circ$ Each scan was evaluated for the primary and nodal lesions according to the NI-RADS lexicon (Table 1). The patient was categorized according to the highest NI-RADS score if multiple lesions were detected. The NI-RADS reporting template was used to evaluate and document the primary and nodal lesions $[7,8,12,13]$. For the primary lesion, the risk categories were NIRADS 1 (no evidence of recurrence) in form of soft tissue distortion, mucosal edema, or post-radiation diffuse linear mucosal enhancement (Figs. 1 and 2), NI-RADS 2 (low suspicion of recurrence) in form of ill-defined deep non-mass like lesion, focal mucosal enhancement, or mild differential enhancement (Figs. 3 and 4) and NI-RADS 3 (high suspicious lesion) with new/enlarging discrete nodule/mass or discrete nodule with differential enhancement (Figs. 5 and 6). For the nodal neck disease, the risk categories were NI-RADS 1 for no enlarging LN, and no new suspicious morphological features (Fig. 1), NI-RADS 2 for growing LN with no morphological abnormality (Fig. 7), and NI-RADS 3 for 
Table 1 NIRADS lexicon radiological features

\begin{tabular}{lll}
\hline NIRADS & Radiological finding (lexicon) & \\
\cline { 2 - 3 } & Primary & Neck (LN) \\
\hline $\begin{array}{l}\text { (No evidence of } \\
\text { recurrence) }\end{array}$ & Soft tissue distortion & No enlarging LN. \\
& Mucosal edema & No new suspicious morphological features \\
II & Diffuse linear mucosal enhancement & \\
$\begin{array}{l}\text { (Low suspicion of } \\
\text { recurrence) }\end{array}$ & Focal mucosal enhancement & Growing LN with no morphological abnormality \\
III & Mild differential enhancement & \\
(High suspicious lesion) & $\begin{array}{l}\text { Discrete nodule/mass (new or enlarged) } \\
\text { enhancement }\end{array}$ & $\begin{array}{l}\text { Growing LN with morphological abnormality (necrosis or extra-nodal } \\
\text { extension) }\end{array}$ \\
\hline
\end{tabular}
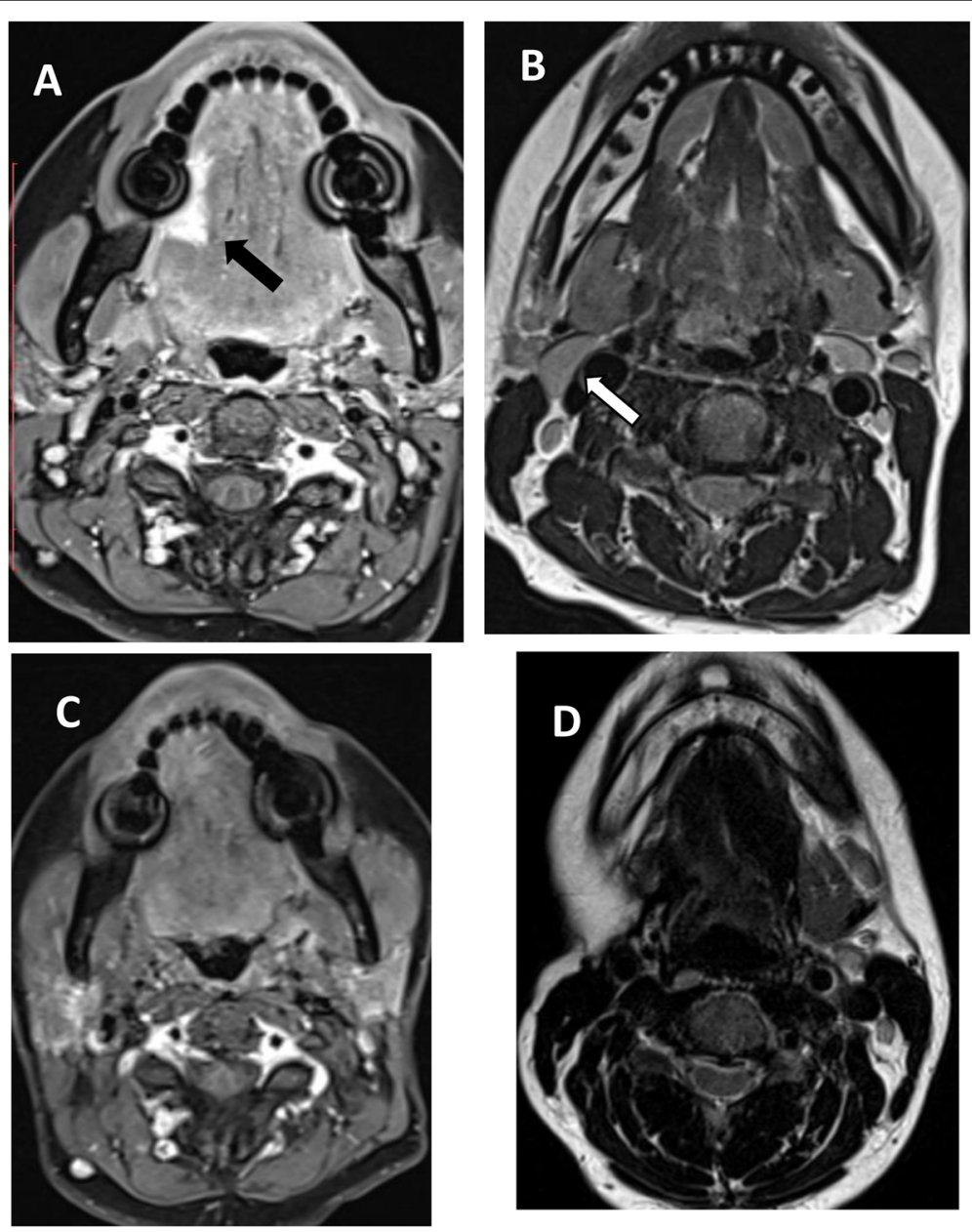

Fig. 1 NI-RADS 1 primary and 1 neck. a, b Upper raw is pretreatment MRI for tongue SCC: a Axial CET1WI shows lateral tongue enhancing soft tissue mass (black arrow). b Axial T2WI shows ipsilateral level II LN (white arrow). c, d Lower raw is post-treatment surveillance MRI reveals resolution of the mass lesion in axial CET1WI (c) and resection of the LN in axial T2WI (d) 

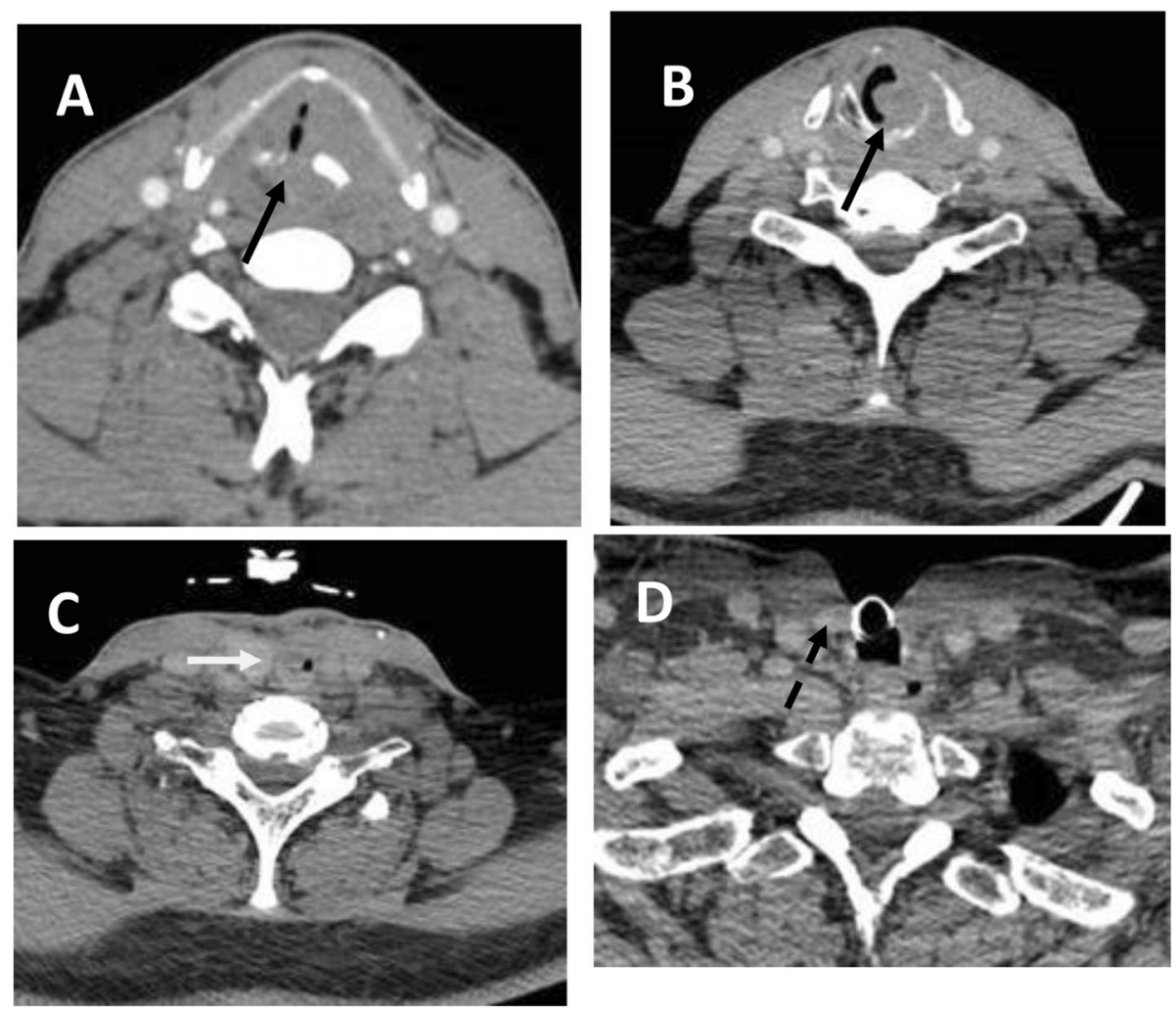

Fig. 2 NI-RADS 1 primary. a, b Glottic and sub-glottic SCC. a, b Axial CE-CT pre-treatment scan shows left glottic and subglottic infiltrating soft tissue mass (black arrows). c, d Post-treatment scan reveals the expected appearance of post total laryngectomy, neopharynx (white arrow), and tracheostomy site (dashed arrow)

growing LN with morphological abnormality (necrosis or extra-nodal extension) (Figs. 7 and 8 ).

$\circ$ The scans reporting were repeated 2 months later by the two readers.

Tumor recurrence was documented in cases of positive biopsy, disease progression in subsequent follow-up scans according to per Response Evaluation Criteria in Solid Tumors [14], or evidence of recurrence on the clinical examination. The lack of tumor recurrence was considered when no evidence of abnormal features in the follow-up scan associated with the absence of recurrence on the clinical examination or negative biopsy.

\section{Statistical analysis}

- Data were fed to the computer and analyzed using Statistical Package for Social Science (IBM Corp. Released 2017. IBM SPSS Statistics for Windows, Version 25.0. Armonk, NY: IBM Corp.).

- Binary variables were used for the morphological and enhancement features of the lesion and the recurrence rate. Ordinal variables were used for the primary and nodal NI-RADS score. Quantitative data were described using range, mean, and standard deviation. Qualitative data were described using the number and percent. The positive predictive value (PPV) of each NI-RADS score was calculated as the number of NI-RADS category positive recurrence divided by the number of NI-RADS category positive and negative recurrence. The negative predictive value (NPV) of each NI-RADS score was calculated as the number of NI-RADS score negative recurrence divided by the number of NI-RADS score positive and negative recurrence. The crosstabulation was performed for estimation of the frequency and percent of each group and for estimation of agreement.

- Fisher's exact test was performed to compare between different groups and the linear by linear association test was used to measure the trend of the ordinal variables. Cramér's $V$ test was performed to measure the effect size of Fisher's exact test with Cramér's $V$ value range from 0 indicating no relationship between the variables and 1 which indicate a perfect relationship. The tests were statistically significant when the $p$ value was less than (0.05). 

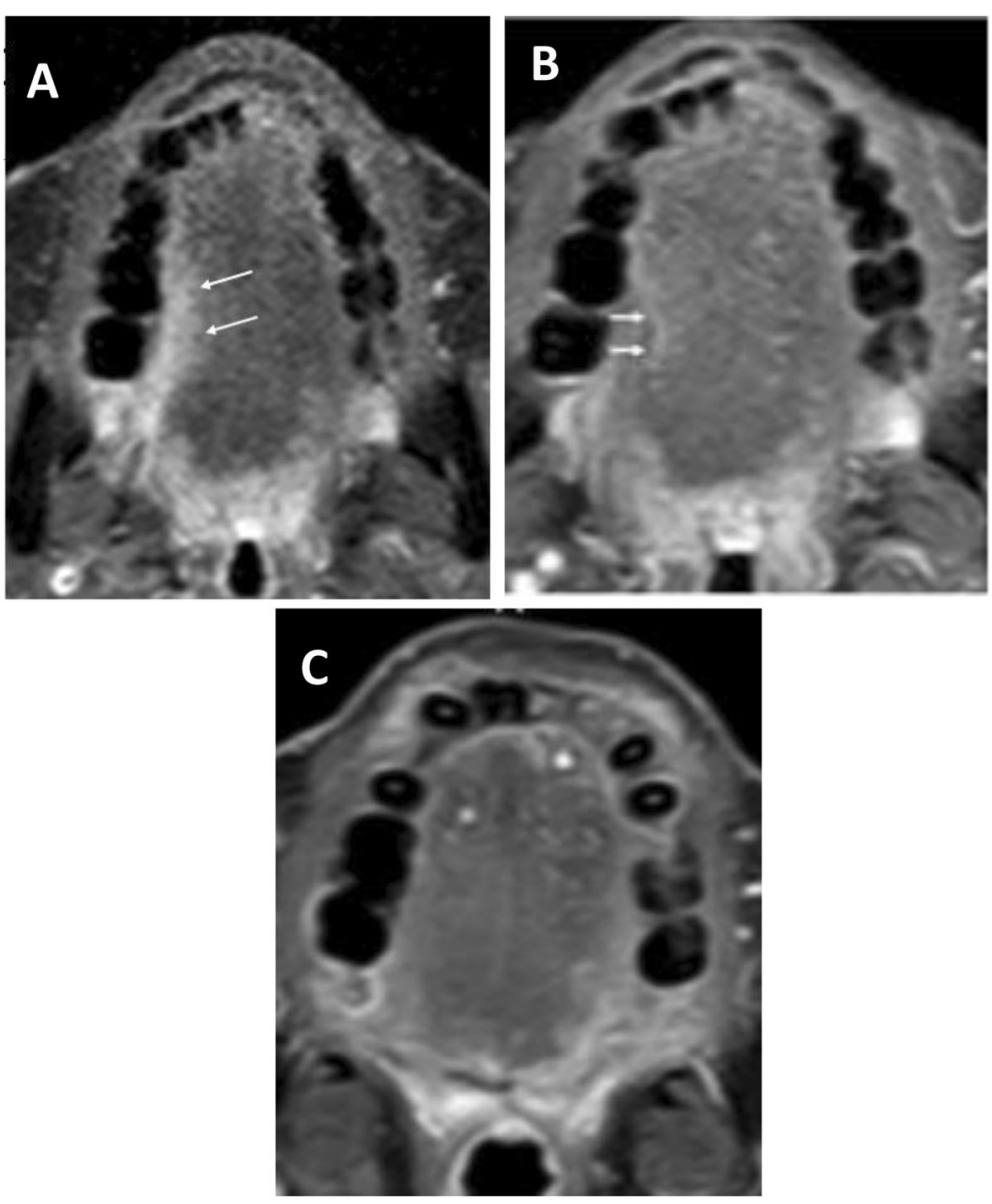

Fig. 3 NI-RADS 2 primary. a Initial post-treatment follow-up axial CET1WI of tongue SCC shows Sheet like ill-defined non-differential enhancement (arrows) at the right lateral aspect of the tongue, with no discrete mass formation. $\mathbf{b}$ Second follow up axial CET1WI shows regressive course of the ill-defined lesion with focal thin linear mucosal enhancement (arrows). c Third follow up axial CET1WI shows resolution of focal mucosal enhancement
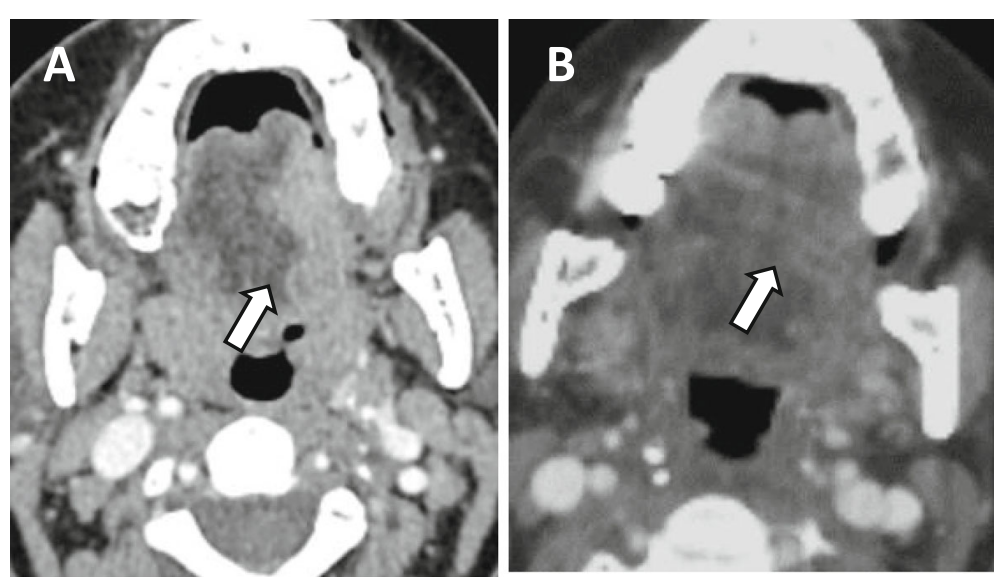

Fig. 4 NI-RADS 2 primary. Tongue SCC. a Axial CECT pre-treatment scan shows left tongue irregular enhancing soft tissue mass (white arrow). b Post-treatment scan reveals regression of the disease with faint non-mass enhancement (white arrow) 

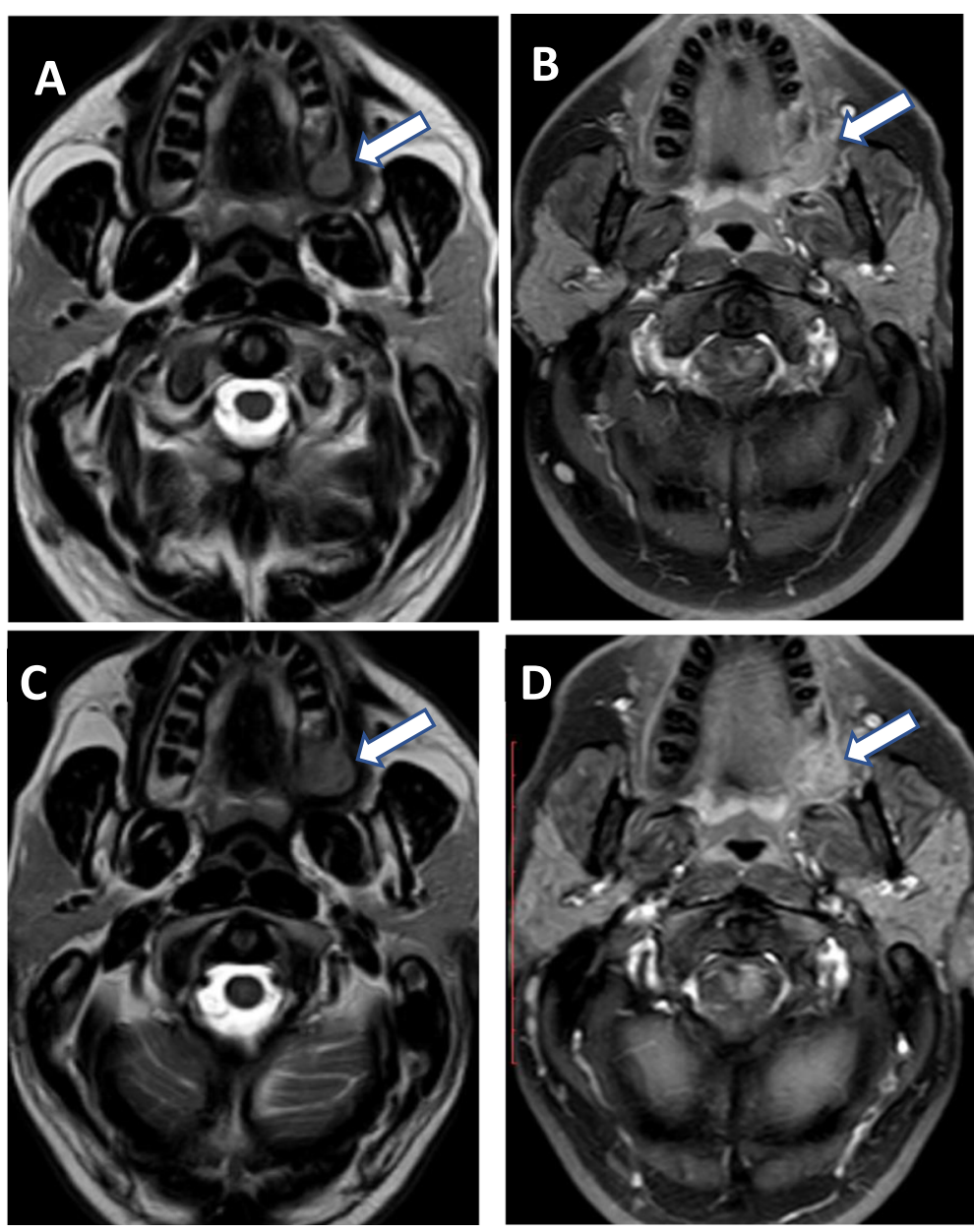

Fig. $5 \mathrm{NI}$-RADS 3 primary. $\mathbf{a}$, b Upper raw is pretreatment MRI for gingival SCC. a axial T2WI. Shows left gingival nodular soft tissue mass (arrow) with post-contrast enhancement in CET1WI (b). c, $\mathbf{d}$ Lower raw is post-treatment MRI shows progression of the gingival mass in axial T2WI (c) and nodular enhancement in CETWI (d)

- A hierarchical logistic regression model was performed to set an estimate of the recurrence rate in NI-RADS 3 primary lesion, the significance of the model, block, and wald statistic were judged at the 5\% level $(p<0.05)$. The first block of the model was reconstructed by adding the morphological variable only, and all statistics were significant, then the second block was reconstructed by adding the enhancement variable, the collinearity and dependency between the two variable were assessed by the percent of regression coefficients variance in association with the smallest eigenvalue, the higher the percent the more the dependency between two variable; in our model, $92 \%$ of region variance of both morphological and enhancement feature was associated with the smallest eigenvalue so the block and wald were insignificant despite that the model was significant. A second logistic region model was reconstructed but with a reverse sequence of variable entrance; the first block included enhancement feature and the morphological feature was added in the second block, all statistic was identical to the first reconstruction model.

\section{Results}

Thirty-four patients with treated laryngeal and oral cavity SCC were examined, 27 (79.4\%) of patients were males and 7 (20.6\%) were females with the mean patient age was $54.5 \pm 2.08$ years (range 19 to 75 years).

Seventy-four scans were examined: 46 (26.2\%) scans for laryngeal lesions and 28 (37.8\%) for oral cavity lesions. The number of first surveillance scans was 33/74 (44.6\%) and the number of follow-up surveillance scan was $41 / 74$ (55.4\%). Fifty-three over seventy-four (71.6\%) scans were CECT and 21/74 (28.4\%) CEMRI.

\section{Performance and agreement of NI-RADS}

The Fisher's exact test was statistically significantly (range of $p$ value was 0.002 to $<0.000$ ) for all primary, 

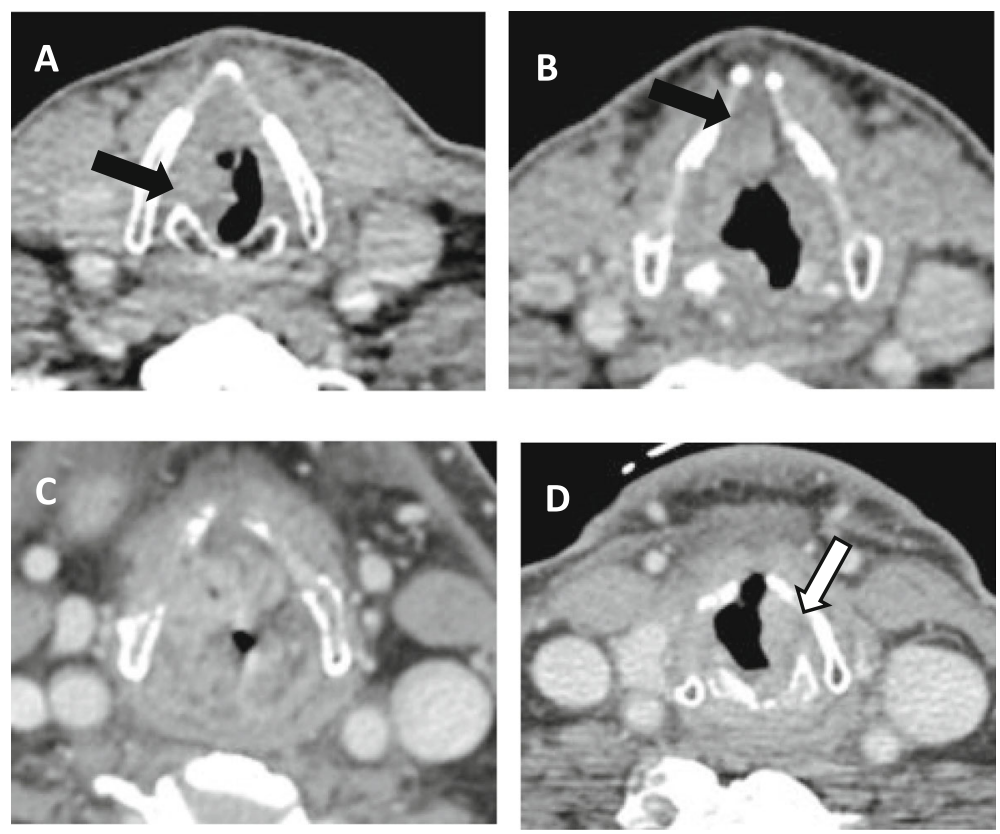

Fig. 6 NI-RADS 3 primary. a, b Upper raw is pretreatment axial CT for laryngeal SCC: show irregular soft tissue mass at the right vocal cord and anterior commissure (black arrow). c, d Lower raw is post-treatment axial CT scan shows diffuse supraglottic soft tissue thickening and enhancement (c), and de-novo left glottic nodular thickening and enhancement (white arrow) (d)

nodal, and combined primary and nodal NI-RADS target lesions, revealing that the recurrence rate was statistically different among the different NI-RADS scores, the Cramér's $V$ also revealed high associating between the NI-RADS score and the recurrence rate (Cramér's $V$ value range 0.486 to 0.841 ). Further analysis revealed a significant trend for recurrence with increased NI-RADS category score (range of $p$ value of the linear by linear association was 0.006 to $<0.000$ ) (Tables 2, 3 and 4).

An almost perfect inter-observed agreement was noted for the laryngeal, oral cavity, all primary lesions, and all nodal lesions with $k=0.859,0.838,0.853$, and 0.840 , respectively.

Both readers revealed an almost perfect intra-observed agreement for the laryngeal, oral cavity, and all primary lesions with reader $1 \mathrm{k}$ was $0.929,0.892$, and 0.915 respectively and reader $2 \mathrm{k}$ was $0.859,0.886$, and 0.872 , respectively.

\section{The overall predictive value of NI-RADS}

The overall NPV of NI-RADS 1 and 2 for the combined primary and nodal lesions were $94.3 \%$ and $74.3 \%$ respectively.

The overall NPV of NI-RAS 1 and 2 for the primary and nodal lesions separately was $90.6 \%$ and $74.1 \%$ for primary lesions and 96.4 and $75 \%$ for nodal lesion, respectively.

The overall PPV of NI-RADS 3 for the combined lesion and the primary and nodal lesions separately was $80.8 \%, 80 \%$, and $81.8 \%$ respectively (Table 2 ).
The predictive value of NI-RADS among laryngeal versus oral cavity lesions subgroup

The number of the recurrent tumor of the combined primary and nodal lesions for the treated oral cavity lesions was significantly different from the that of the treated laryngeal lesion $\left(X^{2}=10.097, p=0.006\right)$, the tumor recurrence was detected in $1 / 25,3 / 15$, and $14 / 16$ for NI-RADS 1, 2, and 3 oral cavity lesions respectively, yet it was proved in $4 / 62,6 / 20$, and $7 / 10$ for NI-RADS 1 , 2 , and 3 laryngeal lesions respectively (Tables 3 and 4).

No significant difference between the number of tumor recurrence of laryngeal and oral cavity primary lesion $(p=0.223)$, The tumor recurrence was detected in $1 / 10,2 / 10$, and $7 / 8$ for oral cavity primary NI-RADS 1 , 2 , and 3 lesions respectively, yet it was proved in $2 / 22$, $5 / 17$, and $5 / 7$ for laryngeal cavity lesions respectively.

The nodal recurrence was seen in $2 / 4,1 / 3$, and $2 / 3$ in laryngeal NI-RADS 1,2 , and 3 respectively, yet it was detected in $0 / 15,1 / 5$, and $7 / 8$ in oral cavity lesions respectively.

\section{Timepoint predictive value of NI-RADS among the first and the follow-up subgroup}

Although no significant difference in the number of the recurrent tumor between the first and the follow-up scans, $X^{2}(13,2)=2.47(p=0.344)$, yet a higher recurrence rate was detected in the NI-RADS 3 follow-up scan in the primary and the combined primary and nodal lesion, with PPV of $83.3 \%$ and $77.8 \%$ respectively, 

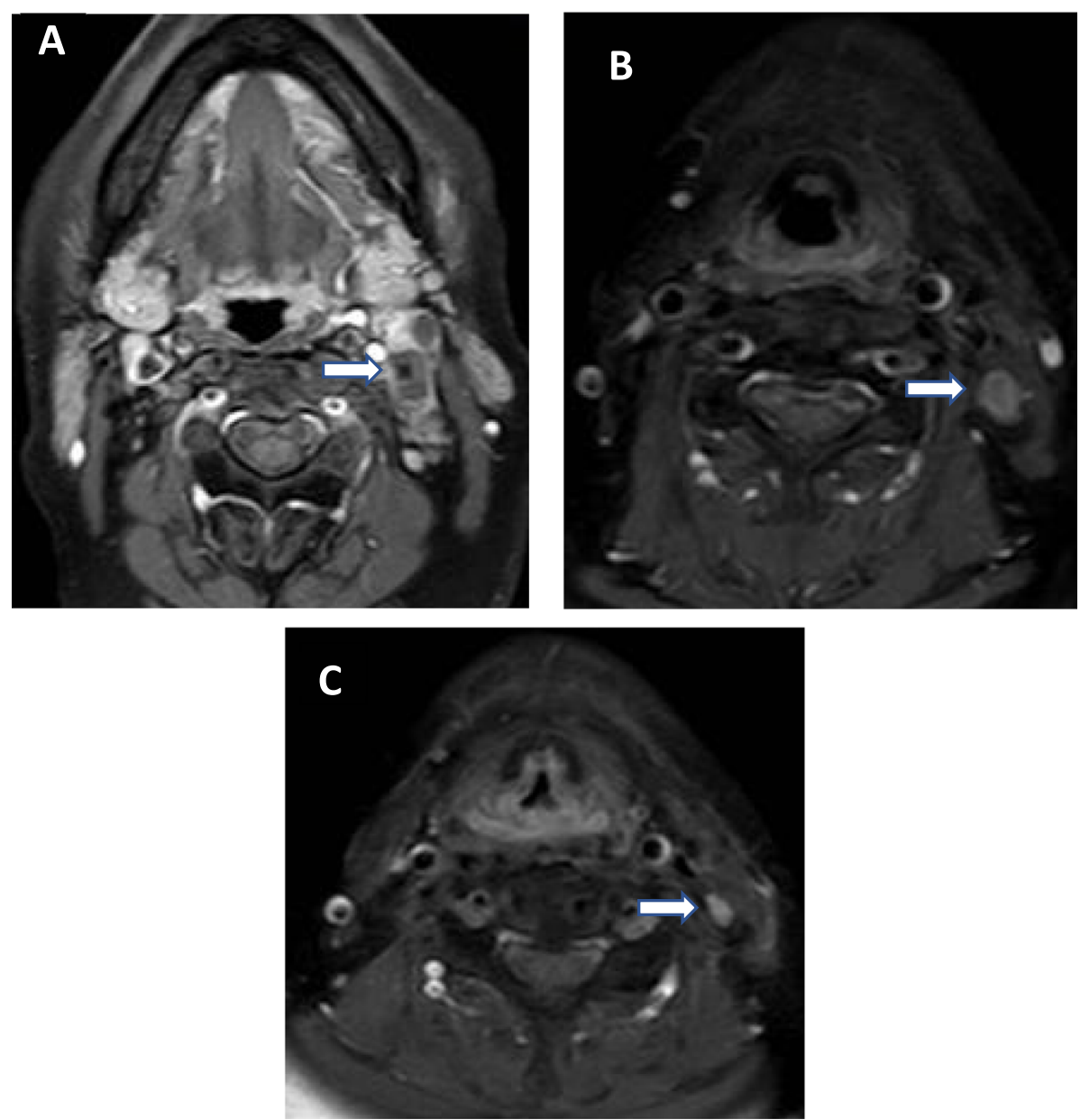

Fig. 7 NI-RADS 2 and 3 neck. a Axial pretreatment CET1WI shows left level II malignant LN with central necrosis (arrow) metastatic from tongue SCC (not shown). b First, a follow-up scan shows residual LN with central breaking down matching NI-RADS 3 neck category. c Second, a followup scan shows regression of the nodal disease with no morphological abnormality in the residual node matching NI-RADS 2 neck

yet the PPV of the first scan was $55.6 \%$ and $70.6 \%$ respectively. On the other hand, the recurrence rate of nodal NI-RADS 3 lesion was higher in the first scan measuring $87.5 \%$ compared to the $66.7 \%$ in the followup scan (Table 5).

The PPV of the follow-up scan NI-RADS 2 nodal lesions was higher than in the first scan $(33.3 \%$ versus $20 \%$ respectively).

Near similar recurrence rate was detected in the first and follow-up scans for NI-RADS 1 lesions and NI-RADS 2 primary and combined primary and nodal lesions.

\section{Logistic region analysis of the primary NI-RADS 3 lesion}

A logistic regression model reconstructed for detection of recurrence rate of the NI-RADS 3 primary lesion revealed that adding either the morphological feature of new or enlarging discrete nodule and mass or the enhancement feature of discrete nodular enhancement significantly influence the model (model, block, and wald $p$
$<0.000$ ), with $b$ value of 2.976 and odd ratio $\{\operatorname{Exp}(\mathrm{B})\}$ of $19.6(95 \% \mathrm{CI}=4.661-82.426)$, the accuracy and the probability of tumor recurrence in NI-RADS 3 if only one feature was added was $82.4 \%$ and $84 \%$ respectively (Table 6). On the other hand, the logistic regression model reconstructed by adding both the morphological and enhancement features together revealed similar value for both features and insignificant block despite significant model which was attributed to the collinearity and dependency between both features (Table 7).

\section{Discussion}

HNSCC is one of the major medical causes of mortality and morbidity worldwide [15]. The incidence of HNSCC varies among different geographical regions according to the prevalence of risk factors [16]. The response to the surgical and radio-chemotherapy treatment is an important prognostic and predictive factor in patient survival $[17,18]$. The distorted anatomy and soft tissue fibrosis occurring after HNSC 


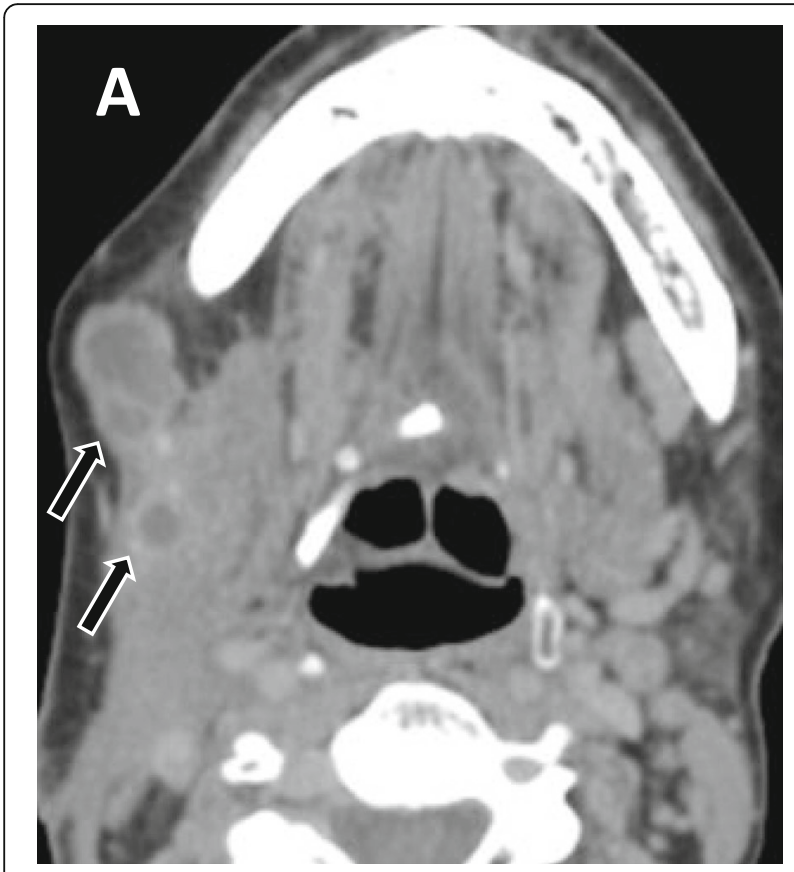

Fig. 8 NI-RADS 3 neck. a Axial CECT post-treatment scan shows necrotic LNs (arrow) metastatic from operated palatal carcinoma (not shown)
$\mathrm{C}$ treatment complicating the scanned image finding and may mimic tumor recurrence which represents a challenge for precise and clear reporting, confusing the clinician, especially when it misses the recommended further surveillance.

The American College of Radiology has developed and updated a structured reporting algorism named Reporting and Data system which was first applied to breast, later on, several systems were developed for different body region as liver, thyroid, prostate, coronary artery, ovarian, colon, and recently head and neck. Each system is formed of imaging lexicon and features with reporting template and algorithm for further management $[8,13,19,20]$.

The NI-RADS scoring system was designed based upon the available literature evidence, best available practices and experience, multidisciplinary consensus, and the biopsy result of the suspicious lesion. This scoring system provided a standardized nomenclature for reporting patients with treated HNSCC and guiding their further management $[6,7,9]$, it was originally developed for reporting the CECT and positron emission tomography (PET) in treated HNSCC patients, the CEMRI was easily adapted to the NI-RADS.

To our knowledge, two previous studies have evaluated the reliability of the NI-RADS, and they revealed an almost perfect interobserver agreement for assigning NIRADS categories [7, 12], on the other hand, few studies have evaluated either the negative or the positive predictive value of the NI-RADS categories in either the first or the follow-up surveillance imaging [11, 12, 21], yet in the current study, we evaluated the NPV of the

Table 2 Predictive value of the overall primary and nodal neck lesions according to NI-RADS categories

\begin{tabular}{|c|c|c|c|c|c|c|c|c|}
\hline \multirow[t]{2}{*}{ NI-RADS } & \multicolumn{2}{|l|}{ Recurrence } & \multicolumn{2}{|c|}{$\begin{array}{l}\text { Fisher's exact } \\
\text { test }\end{array}$} & \multicolumn{2}{|c|}{ Cramér's $(V)$} & \multicolumn{2}{|c|}{$\begin{array}{l}\text { Linear by } \\
\text { linear } \\
\text { association }\end{array}$} \\
\hline & Positive recurrence (PPV\%) & Negative recurrence rate (NPV\%) & Value & $P$ & Value & $P$ & Value & $P$ \\
\hline Primary lesion & & & 23.12 & $<0.000$ & 0.577 & $<0.000$ & 21.64 & $<0.000$ \\
\hline NI-RADS 1 & $3 / 32(9.4 \%)$ & 29/32 (90.6\%) & & & & & & \\
\hline NI-RADS 2 & $7 / 27(25.9 \%)$ & 20/27 (74.1\%) & & & & & & \\
\hline NI-RADS 3 & $12 / 15(80 \%)$ & $3 / 15(20 \%)$ & & & & & & \\
\hline Total & 22/74 (29.7\%) & $52 / 74(70.3 \%)$ & & & & & & \\
\hline LN lesion & & & 31.28 & $<0.000$ & 0.726 & $<0.000$ & 37.1 & $<0.000$ \\
\hline NI-RADS 1 & $2 / 55(3.6 \%)$ & $53 / 55(96.4 \%)$ & & & & & & \\
\hline NI-RADS 2 & $2 / 8(25 \%)$ & $6 / 8(75 \%)$ & & & & & & \\
\hline NI-RADS 3 & 9/11 (81.8\%) & $2 / 11(18.2 \%)$ & & & & & & \\
\hline Total & 13/74 (17.6\%) & $61 / 74(82.4 \%)$ & & & & & & \\
\hline Combined primary and LN & & & 57.06 & $<0.000$ & 0.650 & $<0.000$ & 57.96 & $<0.000$ \\
\hline NI-RADS 1 & $5 / 87(5.7 \%)$ & 82/87 (94.3\%) & & & & & & \\
\hline NI-RADS 2 & $9 / 35(25.7 \%)$ & 26/35 (74.3\%) & & & & & & \\
\hline NI-RADS 3 & 21/26 (80.8\%) & 5/26 (19.2\%) & & & & & & \\
\hline Total & $35 / 148$ (23.6\%) & 113/148 (76.4\%) & & & & & & \\
\hline
\end{tabular}


Table 3 Predictive value of the oral cavity SCC primary and nodal neck lesions according to NI-RADS categories

\begin{tabular}{|c|c|c|c|c|c|c|c|c|}
\hline \multirow[t]{2}{*}{ NI-RADS } & \multicolumn{2}{|l|}{ Recurrence } & \multicolumn{2}{|c|}{$\begin{array}{l}\text { Fisher's exact } \\
\text { test }\end{array}$} & \multicolumn{2}{|c|}{ Cramér's $(V)$} & \multicolumn{2}{|c|}{$\begin{array}{l}\text { Linear by } \\
\text { linear } \\
\text { association }\end{array}$} \\
\hline & Positive recurrence (PPV\%) & Negative recurrence rate (NPV\%) & Value & $P$ & Value & $P$ & Value & $P$ \\
\hline Primary lesion & & & 12.31 & 0.002 & 0.689 & 0.001 & 10.6 & 0.001 \\
\hline NI-RADS 1 & $1 / 10(10 \%)$ & $9 / 10(90 \%)$ & & & & & & \\
\hline NI-RADS 2 & $2 / 10(20 \%)$ & $8 / 10(80 \%)$ & & & & & & \\
\hline NI-RADS 3 & $7 / 8(87.5 \%)$ & $1 / 8(12.5 \%)$ & & & & & & \\
\hline Total & 10/28 (35.7\%) & 18/28 (64.3\%) & & & & & & \\
\hline \multicolumn{9}{|l|}{ LN lesion } \\
\hline NI-RADS 1 & 0/15 (0\%) & 15/15 (100\%) & 18.01 & $<0.000$ & 0.841 & $<0.000$ & 18.01 & $<0.000$ \\
\hline NI-RADS 2 & $1 / 5(20 \%)$ & $4 / 5(80 \%)$ & & & & & & \\
\hline NI-RADS 3 & $7 / 8(87.5 \%)$ & $1 / 8(12.5 \%)$ & & & & & & \\
\hline Total & $8 / 28(28.6 \%)$ & $20 / 28(71.4 \%)$ & & & & & & \\
\hline Combined primary and LN & & & 32.42 & $<0.000$ & 0.763 & $<0.000$ & 28.76 & $<0.000$ \\
\hline NI-RADS 1 & $1 / 25(4 \%)$ & $24 / 25(96 \%)$ & & & & & & \\
\hline NI-RADS 2 & $3 / 15(20 \%)$ & 12/15 (80\%) & & & & & & \\
\hline NI-RADS 3 & $14 / 16(87.5 \%)$ & $2 / 16(12.5 \%)$ & & & & & & \\
\hline Total & 18/56 (32.1\%) & $38 / 56$ (67.9\%) & & & & & & \\
\hline
\end{tabular}

NI-RADS 1 and 2 lesions and the PPV of the NI-RADS 3 lesion. The predictive value was calculated in two of the laryngeal and oral cavity SCC subsites separately and in combination including the first, the follow-up, and the overall scans, also, we analyzed the primary NI-
RADS 3 lesion lexicon using a logistics regression module for the morphological and enhancement features.

In the current study, the surveillance program of the treated laryngeal and oral cavity SCC was performed with CECT and CEMRI, the choice of either imaging

Table 4 Predictive value of the laryngeal SCC primary and nodal neck lesions according to NI-RADS categories

\begin{tabular}{|c|c|c|c|c|c|c|c|c|}
\hline \multirow[t]{2}{*}{ NI-RADS } & \multicolumn{2}{|l|}{ Recurrence } & \multicolumn{2}{|c|}{$\begin{array}{l}\text { Fisher's exact } \\
\text { test }\end{array}$} & \multicolumn{2}{|c|}{ Cramér's $(V)$} & \multicolumn{2}{|c|}{$\begin{array}{l}\text { Linear by } \\
\text { linear } \\
\text { association }\end{array}$} \\
\hline & Positive recurrence (PPV\%) & Negative recurrence rate (NPV\%) & Value & $P$ & Value & $P$ & Value & $P$ \\
\hline Primary lesion & & & 9.90 & 0.005 & 0.486 & 0.003 & 10.06 & 0.002 \\
\hline NI-RADS 1 & $2 / 22(9.1 \%)$ & $20 / 22(90.9 \%)$ & & & & & & \\
\hline NI-RADS 2 & $5 / 17(29.4 \%)$ & 12/17 (70.6\%) & & & & & & \\
\hline NI-RADS 3 & $5 / 7(71.4 \%)$ & $2 / 7(28.6 \%)$ & & & & & & \\
\hline Total & $12 / 46(26.1 \%)$ & $34 / 46(73.9 \%)$ & & & & & & \\
\hline LN lesion & & & 9.48 & 0.012 & 0.524 & $<0.012$ & 12.33 & 0.006 \\
\hline NI-RADS 1 & $2 / 40(5 \%)$ & $38 / 40(95 \%)$ & & & & & & \\
\hline NI-RADS 2 & $1 / 3(33.3 \%)$ & $2 / 3(66.7 \%)$ & & & & & & \\
\hline NI-RADS 3 & $2 / 3(66.7 \%)$ & $1 / 3(33.3 \%)$ & & & & & & \\
\hline Total & $5 / 46(10.9 \%)$ & 41/46 (89.1\%) & & & & & & \\
\hline Combined primary and LN & & & 21.61 & $<0.000$ & 0.525 & $<0.000$ & 24.5 & $<0.000$ \\
\hline NI-RADS 1 & $4 / 62(6.5 \%)$ & $58 / 62(93.5 \%)$ & & & & & & \\
\hline NI-RADS 2 & $6 / 20(30 \%)$ & 14/20 (70\%) & & & & & & \\
\hline NI-RADS 3 & 7/10 (70\%) & $3 / 10(30 \%)$ & & & & & & \\
\hline Total & 17/92 (18.5\%) & 75/92 (81.5\%) & & & & & & \\
\hline
\end{tabular}


Table 5 Recurrence rate of the primary and nodal neck lesions among the first and follow-up scans

\begin{tabular}{|c|c|c|c|c|c|c|}
\hline \multirow[t]{2}{*}{ NI-RADS } & \multicolumn{2}{|l|}{ Primary lesion } & \multicolumn{2}{|l|}{ Nodal lesion } & \multicolumn{2}{|c|}{ Combined primary and nodal lesions } \\
\hline & First scan (\%) & Follow-up scan (\%) & First scan (\%) & Follow-up scan (\%) & First scan (\%) & Follow-up scan (\%) \\
\hline $\mathrm{NI}$-RADS 1 & $0 / 9(0 \%)$ & $1 / 23(4.3 \%)$ & $1 / 20(5 \%)$ & $1 / 35(2.9 \%)$ & $1 / 29(3.4 \%)$ & $2 / 58(3.4 \%)$ \\
\hline NI-RADS 2 & $4 / 15(26.7 \%)$ & $3 / 12(25 \%)$ & $1 / 5(20 \%)$ & $1 / 3(33.3 \%)$ & $5 / 20(25 \%)$ & $4 / 15(26.7 \%)$ \\
\hline NI-RADS 3 & $5 / 9(55.6 \%)$ & $5 / 6(83.3 \%)$ & $7 / 8(87.5 \%)$ & $2 / 3(66.7 \%)$ & 12/17 (70.6\%) & $7 / 9(77.8 \%)$ \\
\hline Total & 9/33 (27.3\%) & 9/41 (22\%) & 9/33 (27.3\%) & 4/41 (9.8\%) & 18/66 (27.3\%) & 13/82 (15.8\%) \\
\hline
\end{tabular}

modality was tailored according to the primary tumor site, the specific clinical question to be answered and the patients' clinical status, considering the lengthy CEMRI examination.

In this study, the post-treatment surveillance scans were assessed according to the NI-RADS, a significant trend for tumor recurrence was noted with increased NI-RADS category.

For both first and follow-up scan, the overall recurrent rate of NI-RADS 1 and 2 primary lesions was 9.4\% and $25.9 \%$ respectively with the NPV of $90.6 \%$ and $74.1 \%$. Krieger et al. [12] reported a slightly lower recurrence rate for primary NI-RADS 1 and 2 lesions with higher NPV of $96.5 \%$ and $81.6 \%$ respectively. The PPV of the NI-RADS 3 primary lesion in our study was $80 \%$ which was higher than the reported value $(59.4 \%)$ seen in the Krieger et al. [12]. This difference is explained in part by the different imaging modality as the surveillance imaging modalities in the Krieger et al. [12] study were $\mathrm{PET} / \mathrm{CT}$ and CECT, yet in the current study, the surveillance imaging was based solely in the cross-sectional examination. Furthermore, Krieger et al. [12] reported much higher PPV for combined primary and nodal NIRADS 3 diagnosed with CECT only, with a recurrence rate measuring $91.7 \%$, near similar result was seen in the current study which revealed primary and nodal lesion recurrence in $88.8 \%$ of patients assigned as NI-RADS 3 category. These data suggest that the CECT and CEMRI are more specific and the metabolic and functional data gained with the PET examination shows an impact on the predictive value of NI-RADS score.

In this work, the recurrence rate of the overall first and follow-up NI-RADS 1 nodal lesion was 3.6\% which was similar to Krieger et al. [12] result. The NPV of nodal NI-RADS 2 was $75 \%$ which was lower than the value seen in the Krieger et al. [12] study who revealed
NPV of $85 \%$. On the other hand, the PPV of nodal NIRADS 3 was $81.8 \%$ which was higher than the value seen in Krieger et al. [12] study (70\%). Different studies will vary at their PPV and NPV according to variable time points of the included studies. In the current study, the PET/CT was not used as a surveillance imaging and the nodal NI-RADS 3 lesions diagnosed solely according to the abnormal morphological features.

As regards the post-treatment first scan, the NPV of NI-RADS 1 and 2 primary lesions were 100\% and 73.3\% respectively. Hsu et al. [11] reported a slightly lower recurrence rate for the post-treatment primary NI-RADS 1 lesion with NPV of $93.6 \%$, yet the NPV of primary NIRADS 2 lesions was higher than our value (88.9\%). The PPV of the first post-treatment primary NI-RADS 3 lesions in our study was $55.6 \%$; a lower value was seen in Hsu et al. [11] who reported PPV of $38.5 \%$.

Although no significant difference among the first and the follow-up recurrence rate, yet the PPV of NI-RADS 3 lesion was higher in the follow-up scan, a similar result was reported by Krieger et al. [12]. The first posttreatment surveillance scan represents a challenge for its interpretation due to the distorted and complex anatomy, in addition to the early post-treatment changes that usually regress in the further follow-up scans.

The PPV of the oral cavity NI-RADS 3 primary lesions was higher than that of laryngeal lesions. Further studies may reveal a difference in the NI-RADS predictive value among different HNSCC subsites. The previous study revealed an almost perfect interobserver agreement in the discrimination of the treated laryngeal and oral cavity primary target lesion [7].

The odd of tumor recurrence for NI-RADS 3 primary lesion was 19.6 times that for NI-RADS 1 and 2 primary lesions. The odd ratio was similar if either the morphological lexicon of new/enlarging discrete nodule/mass or

Table 6 Logistic regression model for NI-RADS 3 primary lesions reconstructed by either the morphological feature of new or enlarging discrete nodule and mass or the enhancement feature of discrete nodular enhancement

\begin{tabular}{|c|c|c|c|c|c|c|c|c|c|c|c|}
\hline & \multirow[t]{2}{*}{$B$} & \multirow{2}{*}{$\begin{array}{l}\text { Exp } \\
(B)\end{array}$} & \multicolumn{2}{|c|}{$95 \%$ C.I. for $\operatorname{Exp}(B)$} & \multirow[t]{2}{*}{ Accuracy } & \multirow[t]{2}{*}{ Probability } & \multicolumn{2}{|l|}{$P$} & \multicolumn{2}{|l|}{ Wald } & \multirow{2}{*}{$\begin{array}{l}\text { Nagelkerke } \\
R \text { square }\end{array}$} \\
\hline & & & Lower & Upper & & & Model & Block & Value & $P$ & \\
\hline Constant & -1.589 & 0.204 & & & & & & & 20.98 & $<0.000$ & \\
\hline Feature $^{a}$ & 2.976 & 19.600 & 4.661 & 82.426 & $82.4 \%$ & $84 \%$ & $<0.000$ & $<0.000$ & 16.49 & $<0.000$ & 0.356 \\
\hline
\end{tabular}

Both features showed similar value 
Table 7 Logistic regression model for NI-RADS 3 primary lesions reconstructed by both the morphological and enhancement features

\begin{tabular}{|c|c|c|c|c|c|c|c|c|c|c|c|}
\hline \multirow[t]{2}{*}{ 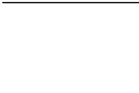 } & \multirow[t]{2}{*}{ B } & \multirow{2}{*}{$\begin{array}{l}\text { Exp } \\
(B)\end{array}$} & \multicolumn{2}{|c|}{ 95\% C.I. for $\operatorname{Exp}(B)$} & \multirow[t]{2}{*}{ Accuracy } & \multirow[t]{2}{*}{ Probability } & \multicolumn{2}{|l|}{$P$} & \multicolumn{2}{|l|}{ Wald } & \multirow{2}{*}{$\begin{array}{l}\text { Nagelkerke } \\
R \text { square }\end{array}$} \\
\hline & & & Lower & Upper & & & Model & Block & Value & $P$ & \\
\hline Constant & -1.675 & 0.187 & & & & & & & 21.858 & $<0.000$ & \\
\hline Feature $^{a}$ & 1.688 & 5.408 & 0.650 & 45.027 & $82.4 \%$ & $84 \%$ & $<0.000$ & 0.131 & 2.436 & 0.119 & 0.388 \\
\hline
\end{tabular}

${ }^{\mathrm{a}}$ Both features showed similar value

the enhancement lexicon of discrete nodular enhancement was detected. Future studies may highlight the contribution of a single NI-RADS lexicon in the recurrence rate. Abdelaziz et al. [7] revealed significant interobserver agreement for detection of discrete nodule/ mass and for excluding tissue enhancement.

The accuracy of NI-RADS 3 for lesion recurrence was $80.8 \%$, yet there was still $19.2 \%$ lesions in which biopsy recommendation was not balanced by the positive recurrence, further evaluation of the enhancement pattern of the NI-RADS lesion with the dynamic contrast-enhanced MRI, MRI perfusion, and diffusion which was not involved in the NI-RADS lexicon may provide a precise characterization of NI-RADS 2 and 3 lesions, and differentiating between tumor recurrence and post-treatment soft tissue injury which mimics residual tumor.

Finally, the NI-RADS provide a relevant skeleton for further management recommendation based on the evidence-based recurrence rate of each NI-RADS category, assuring patient with NI-RADS 1 and 2 categories, and provide a reasonable reason for biopsy in NIRADS 3 lesion.

\section{Limitation}

Some limitations were encompassed in our study. First, the NI-RADS 3 lesions represented a small percentage of overall NI-RADS lesions in this limited number study and the major NI-RADS lesions were categorized as NIRADS 1 (17.6\% (26/148) versus 58.8\% (87/148) respectively), yet our percentage was greater than previously published figures where NI-RADS 3 lesions represented only $4.8 \%$ and $5.2 \%[11,12]$. Second, we did not scrutinize all HNSCC subsites collectively. The investigation of two different subsites separately highlights the difference in the NI-RADS categories and recurrence rate among them. Further study may provide data for each subsite. Third, in this study, we did not correlate the NI-RADS categories to the tumor staging, prevalence of human papillomavirus infection, and the type of management either surgical, radiotherapy, chemotherapy, or combination management. Finally, the combination of CEMRI and CECT scans in our study, instead of investigating each modality separately, yet the previous study also involved the PECT/CT and CECT collectively [11].

\section{Conclusion}

A significant trend for tumor recurrence was noted with higher NI-RADS categories. The NPV of NI-RADS 1 was $94.3 \%$, and patient reassurance with routine surveillance is the optimal next step. Although the NPV of NI-RADS 2 was $80 \%$, yet close follow-up with or without PET/CT is the preferred management. The PPV of NI-RADS 3 was 88.5\%; the target biopsy is the optimal next step. The NIRADS performance may differ among the HNSCC primary tumor subsite. The detection of new/enlarging discrete nodules or discrete nodular enhancement equally categorizes the primary lesion as NI-RADS 3.

\section{Abbreviations}

NI-RADS: Neck imaging reporting and data system; SCC: Squamous cell carcinoma; CEMRI: Contrast-enhanced magnetic resonance imaging; CECT: Contrast-enhanced computed tomography; NPV: Negative predictive value; PPV: Positive predictive value; HNSCC: Head and neck squamous cell carcinoma; ACR: American College of Radiology; PET: Positron emission tomography

\section{Acknowledgements}

Not applicable

\section{Authors' contributions}

AS: Results and statistics, manuscript writing. TT: Idea of the research, revising the manuscript. MM: Idea of the research, collecting cases. All authors read and approved the final manuscript.

Funding

No funds, sponsorship, or financial support to be disclosed.

\section{Availability of data and materials}

The datasets used and/or analyzed during the current study are available from the corresponding author on reasonable request.

\section{Ethics approval and consent to participate}

This study was approved by the Research Ethics Committee of the Faculty of Medicine at Ain shams University in Egypt (FWA 000017585); Reference Number of approval: R67/2020. The requirement for written consent was waived in this retrospective study.

\section{Consent for publication}

Not applicable.

\section{Competing interests}

The authors declare that they have no competing interests.

Received: 28 September 2020 Accepted: 22 November 2020 Published online: 30 November 2020

\section{References}

1. Bourhis J, Sire C, Graff P et al (2012) Concomitant chemo-radiotherapy versus acceleration of radiotherapy with or without concomitant chemotherapy in locally advanced head and neck carcinoma (GORTEC 9902): an open-label phase 3 randomised trial. Lancet Oncol 13(2):145-153 
2. Beswick DM, Gooding WE, Johnson JT et al (2012) Temporal patterns of head and neck squamous cell carcinoma recurrence with positron-emission tomography/computed tomography monitoring. Laryngoscope 122(7): 1512-1517

3. Posner MR, Hershock DM et al (2007) Cisplatin and fluorouracil alone or with docetaxel in head and neck cancer. N Engl J Med 357:1705-1715

4. Chang JH, Wu CC, Yuan KS et al (2017) Locoregionally recurrent head and neck squamous cell carcinoma: incidence, survival, prognostic factors, and treatment outcomes. Oncotarget 8(33):55600

5. Gupta B, Johnson NW, Kumar N et al (2016) Global epidemiology of head and neck cancers: a continuing challenge. Oncology 91(1):13-23

6. Aiken A, Farley A, Baugnon $\mathrm{K}$ et al (2016) Implementation of a novel surveillance template for head and neck cancer: neck imaging reporting and data system (NI-RADS). J Am Coll Radiol 13(6):743-746

7. Abdelaziz TT, Abdel Razk AAK, Ashour MMM et al (2020) Interreader reproducibility of the neck imaging reporting and data system (NI-RADS) lexicon for the detection of residual/recurrent disease in treated head and neck squamous cell carcinoma (HNSCC). Cancer Imaging 20:61

8. Aiken AH, Rath TJ, Anzai Y et al (2018) ACR neck imaging reporting and data systems (NI-RADS): a white paper of the ACR NI-RADS Committee. $J$ Am Coll Radiol 15(8):1097-1108

9. Dodd GD III, Allen B Jr, Birzniek D et al (2015) Reengineering the radiology enterprise: a summary of the 2014 Intersociety Committee Summer Conference. J Am Coll Radiol 12(3):28-234

10. Aiken A, Hudgins P (2018) Neck imaging reporting and data system. Magn Reson Imaging Clin N Am 26(1):51-62

11. Hsu D, Chokshi FH, Hudgins PA et al (2019) Predictive value of first posttreatment imaging using standardized reporting in head and neck cancer. Otolaryngol Head Neck Surg 161(6):978-985

12. Krieger $D$, Hudgins $P$, Nayak $G$ et al (2017) Initial performance of NI-RADS to predict residual or recurrent head and neck squamous cell carcinoma. AJNR Am J Neuroradiol 38(6):1193-1199

13. Abdel Razek AAK, Abdelaziz TT (2020) Neck imaging reporting and data system: what does radiologist want to know? J Comput Assist Tomogr 44(4):527-532

14. Schwartz LH, Litière S, de Vries E et al (2016) RECIST 1.1-update and clarification: from the RECIST committee. Eur J Cancer 62:132-137

15. Kang H-S, Roh J-L, Lee S-W et al (2016) Noncancer-related health events and mortality in head and neck cancer patients after definitive radiotherapy: a prospective study. Medicine 95(19):e3403. https://doi.org/10.1097/md. 0000000000003403.

16. Parasher AK, Abramowitz M, Weed D et al (2014) Ethnicity and clinical outcomes in head and neck cancer: an analysis of the SEER database. J Racial Ethn Health Disparities 1:267-274

17. Martens RM, Noij DP, Ali M et al (2019) Functional imaging early during (chemo)radiotherapy for response prediction in head and neck squamous cell carcinoma; a systematic review. Oral Oncol 88:75-83

18. Subramaniam RM, Lowe VJ (2007) Squamous cell carcinoma: restaging and response to therapy. PET Clin 2:481-487

19. An JY, Unsdorfer KML, Weinreb JC (2019) BI-RADS, C-RADS, CAD-RADS, LIRADS, Lung-RADS, NI-RADS, O-RADS, PI-RADS, TI-RADS: reporting and data systems. Radiographics 39(5):1435-1436

20. Azab EA, Abdelrahman AS, Ibrahim MEA (2019) A practical trial to use Thyroid Imaging Reporting and Data System (TI-RADS) in differentiation between benign and malignant thyroid nodules. Egypt J Radiol Nucl Med 50:17

21. Wangaryattawanich P, Branstetter B, Hughes M et al (2018) Negative predictive value of NI-RADS category 2 in the first posttreatment FDG-PET/ $\mathrm{CT}$ in head and neck squamous cell carcinoma. AJNR Am J Neuroradiol 39(10):1884-1888

\section{Publisher's Note}

Springer Nature remains neutral with regard to jurisdictional claims in published maps and institutional affiliations.

\section{Submit your manuscript to a SpringerOpen ${ }^{\circ}$ journal and benefit from:}

- Convenient online submission

- Rigorous peer review

- Open access: articles freely available online

- High visibility within the field

- Retaining the copyright to your article

Submit your next manuscript at $\boldsymbol{\nabla}$ springeropen.com 